\title{
Laboratory Assessment of a Gravity-Fed Ultrafiltration Water Treatment Device Designed for Household Use in Low-Income Settings
}

\author{
Thomas Clasen,* Jaime Naranjo, Daniel Frauchiger, and Charles Gerba \\ Department of Infectious and Tropical Diseases, London School of Hygiene and Tropical Medicine, London, England; \\ Department of Soil, Water and Environmental Science, University of Arizona, Tucson, Arizona; and \\ Vestergaard Frandsen S.A., Lausanne, Switzerland
}

\begin{abstract}
Interventions to improve water quality, particularly when deployed at the household level, are an effective means of preventing endemic diarrheal disease, a leading cause of mortality and morbidity in the developing world. We assessed the microbiologic performance of a novel water treatment device designed for household use in low-income settings. The device employs a backwashable hollow fiber ultrafiltration cartridge and is designed to mechanically remove enteric pathogenic bacteria, viruses, and protozoan cysts from drinking water without water pressure or electric power. In laboratory testing through $20,000 \mathrm{~L}$ ( $110 \%$ of design life) at moderate turbidity (15 nephelometric turbidity unit [NTU]), the device achieved $\log _{10}$ reduction values of 6.9 for Escherichia coli, 4.7 for MS2 coliphage (proxy for enteric pathogenic viruses), and 3.6 for Cryptosporidium oocysts, thus exceeding levels established for microbiological water purifiers. With periodic cleaning and backwashing, the device produced treated water at an average rate of $143 \mathrm{~mL} / \mathrm{min}(8.6 \mathrm{~L} / \mathrm{hour})$ (range 293 to $80 \mathrm{~mL} / \mathrm{min}$ ) over the course of the evaluation. If these results are validated in field trials, the deployment of the unit on a wide scale among vulnerable populations may make an important contribution to public health efforts to control intractable waterborne diseases.
\end{abstract}

\section{INTRODUCTION}

Unsafe drinking water is a leading cause of preventable disease, particularly among young children in developing countries. ${ }^{1}$ Waterborne pathogens, including a variety of viral, bacterial, and protozoan agents, account for much of the estimated 4 billion cases and 1.8 million deaths from endemic diarrheal disease each year. ${ }^{2}$ Among children $<5$ years of age in developing countries, diarrheal disease accounts for $17 \%$ of all deaths. ${ }^{2}$ Microbiologically contaminated water also contributes to the heavy burden of disease associated with cholera, typhoid, paratyphoid, hepatitis, poliomyelitis, and gastroenteritis. Low-income populations are particularly at risk of such diseases because of the unavailability of safe water and sanitation. ${ }^{3}$

Interventions to treat and maintain the microbiological quality of water at the household level are a promising alternative for households without access to a reliable supply of safe drinking water. In many settings, both rural and urban, household-based water treatment has been shown to be more effective in reducing endemic diarrhea than conventional treatment at the source or point of distribution. ${ }^{4,5}$ Among household-based water treatment interventions, filters have been shown to be particularly protective against diarrheal disease. ${ }^{5}$ Household-based water treatment has also been shown to be highly cost-effective. ${ }^{6}$

Reaching vulnerable populations at scale with an effective, low-cost, long-lasting, and otherwise suitable water treatment device has been particularly challenging. ${ }^{7}$ Although promoters of chlorination, filtration, solar disinfection, and flocculation/ disinfection reported increased coverage and uptake of household water treatment among low-income populations by $26 \%$ in 2006 and 2007 to 19 million people, this represents less than $2 \%$ of the persons without access to improved water supplies. ${ }^{7,8}$ It is even a smaller portion of those whose water is microbiologically

* Address correspondence to Thomas Clasen, Disease Control and Vector Biology Unit, Department of Infectious and Tropical Diseases, London School of Hygiene and Tropical Medicine, Keppel St., London WC1E 7HT, UK. E-mail: thomas.clasen@1shtm.ac.uk safe. ${ }^{8}$ Although dozens of products have been developed and tested, ${ }^{9}$ few meet the microbiologic performance levels for reductions in bacteria, viruses, and protozoa established by the U.S. Environmental Protection Agency (USEPA) ${ }^{10}$ and incorporated into National Sanitation Foundation (NSF)/ American National Standards Institute (ANSI) standards, and none of these has secured widespread coverage among lowincome populations. Although some microfilters have been embraced over chemical disinfectants because of their capacity to improve water aesthetics, most such devices also present disadvantages, including comparatively high up-front cost, low output, limited longevity, and susceptibility to premature clogging and breakage.

Vestergaard Fransen S.A. (Lausanne, Switzerland), the main producer of pipe filters for the Guinea Worm Eradication Initiative and long-lasting insecticide-treated nets (LLINs) for malaria prevention, has developed a microbiological water purifier known as "LifeStraw Family." The device is specifically designed to build on the company's experience in rapidly scaling up the production and distribution of personal and household-based environmental health interventions among vulnerable, low-income populations. This work reports on laboratory testing of the microbiologic effectiveness, flow rate, and longevity of this new treatment unit.

\section{THE WATER TREATMENT SYSTEM}

The LifeStraw Family is a fully-integrated, gravity-fed, pointof-use microbial water treatment system intended for routine use in low-income settings. To meet the needs of the most vulnerable populations, it was designed to operate without electricity or other power and without a piped-in water supply. The unit was also designed to treat water of unknown microbiologic quality, and thus meet internationally recognized levels for microbiological water purifiers. It was also designed to operate under heavier levels of turbidity that may characterize water in such settings, especially during rainy seasons.

The microbiologic barrier consists of a $26-\mathrm{cm}-$ long $\times 3-\mathrm{cm}-$ diameter plastic cylindrical cartridge containing a number of hollow fibers with a $20-\mathrm{nm}$ pore size. Although the cartridge is 
potentially suitable for tabletop and other configurations, we tested a configuration designed for mass distribution and use in settings that do not necessarily have surfaces for tabletop units (Figure 1). Source water is introduced into the system by dipping the $2.5 \mathrm{~L}$ receptacle into an open vessel or pouring water into it if hanging or mounted on a wall. The water passes through a cleanable $27-\mu$ m textile prefilter mounted inside a removal plastic basket inside the receptacle and then through a $1 \mathrm{~m}$ length of 12-mm-diameter plastic tubing filling the cartridge. A slow-eluting solid chlorine tablet can be installed in a halogen chamber at the receptacle to help prevent biofilm, but was not included in this assessment to avoid any biocidal action that might be attributable to the disinfectant. When the side tap is opened, water passes through the walls of the hollow fiber membrane bundle and out the tap, mechanically removing microbes and other suspended solids greater than $20 \mathrm{~nm}$ in size.

The prefilter is cleaned by removing the prefilter basket from the receptacle and rinsing it in water. The microbial cartridge must also be cleaned from time to time by backwashing it. This is done by closing off the side tap, squeezing the hand pump located on the lower part of the cartridge three times, and opening the cock at the bottom of the cartridge for a few seconds to allow the backwash to flow to waste. The bottom cock is then closed and the unit is ready for use.

The unit is designed to produce $\sim 150 \mathrm{~mL}$ of product water/ minute (9 L/hour) and to last for at least 18,000 L. As it relies on mechanical filtration and not disinfection or adsorption, there is no need for a means of measuring volume of water

\section{LifeStraw ${ }^{\circledR}$ Family}

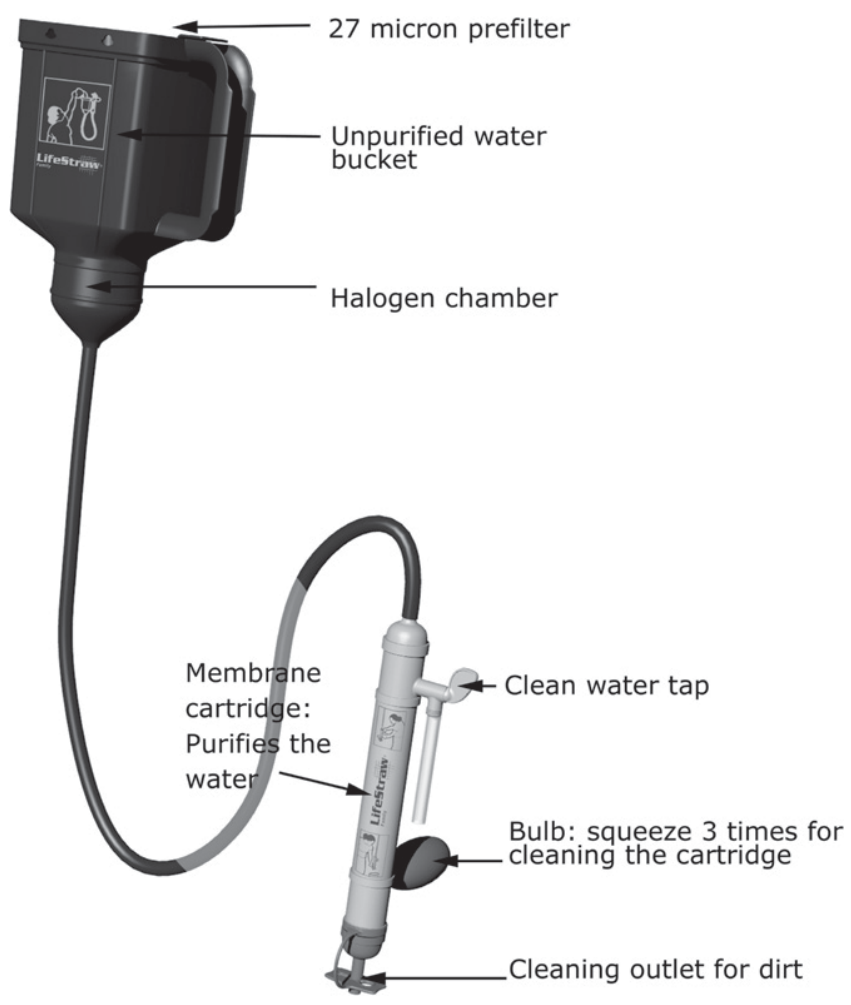

Figure 1. Schematic of device. treated or end of useful life; as long as the device remains intact, water from the tap will be effectively treated. When the flow from the unit cannot be restored to an acceptable rate by prefilter cleaning and cartridge backwashing, the entire unit is intended to be replaced. Assuming a household of five persons, the unit would provide $2 \mathrm{~L}$ of drinking water/person/day for almost 5 years without any replacement parts. In larger quantities, the manufacturer sells this configuration for about US\$20.00. Using the foregoing assumptions, this works out to less than US $\$ 1 /$ person/year. The cost per liter treated would be US\$0.001/L.

\section{METHODS AND MATERIALS}

Setup and test waters. Test methods were based generally on EPA Protocol and Guide Standard for Testing Microbiological Water Purifiers (the "EPA Protocol"). ${ }^{10}$ Three production units of the LifeStraw Family provided by the manufacturer were conditioned in accordance with the manufacturer's instructions with unspiked test water and installed on the bench for testing using apparatus conforming to EPA Protocol (Figure 1). Aging was performed using water based on EPA general test water \#1, except that the turbidity level was increased from 0.1 to 5.0 NTU prescribed by the Protocol to 15 nephelometric turbidity unit (NTU), and the organic carbon level was increased from $0.1-5.0 \mathrm{mg} / \mathrm{L}$ prescribed under the Protocol to $5 \mathrm{mg} / \mathrm{L}$. These harsher conditions were intended to challenge the longevity and flow rate of the device. Microbial challenges were performed using water based on EPA challenge test water \#3, except that the water was maintained at room temperature and not chilled to $4^{\circ} \mathrm{C}$ as prescribed by Protocol. The performance of occlusion devices, such as the LifeStraw Family, is not expected to be impacted by low temperatures, which are known to affect halogen disinfection. The parameters for the test water, including the materials used for adjusting the parameter, are set forth in Table 1.

Test organisms. The test organisms consisted of microbes shown to simulate the range of waterborne pathogens commonly found in untreated water. The bacteria group was represented by Escherichia coli (ATCC \# 25922) spiked into the input water at concentrations of $10^{7}$ to $10^{8}$ colony forming units (CFU)/100 mL. The viral group was represented by malespecific coliphage MS2 (ATCC \#15597-B-1) spiked into the input water at concentrations of $5 \times 10^{8}$ plaque forming units (PFU)/100 mL and inoculated into E. coli (ATCC \# 15597) for assay. The MS2 coliphage has been recognized as a suitable surrogate for enteric viruses for water treatment processes ${ }^{11}$ and point-of-use device testing. ${ }^{12}$ The protozoan cyst group was represented by Cryptosporidium parvum oocysts spiked into the input water at concentrations of $5 \times 10^{6} / \mathrm{L}$.

Microbiologic methods. Escherichia coli was grown overnight in Trypticase Soy broth (Difco, Detroit, MI) at $37^{\circ} \mathrm{C}$ to obtain the organisms in the stationary growth phase. The bacterial cells were pelleted by centrifugation and resuspended in phosphate buffered saline (PBS). This procedure was repeated three times to remove organic matter present in the broth. Bacterial assays were conducted by the membrane filtration method on m-Endo Agar LES (Becton Dickinson, Cockeysville, MD). Appropriate dilutions of influent samples were made in sterile 0.025 M PBS at pH 7.0. A $100 \mathrm{~mL}$ sample of undiluted unit effluent was also assayed. All assays were in triplicate according to Standard Methods. ${ }^{13}$ The MS-2 virus 
TABLE 1

Test water parameters*

\begin{tabular}{|c|c|c|c|}
\hline Parameter & Aging water & Challenge water & Material for adjusting test water characteristics \\
\hline $\mathrm{pH}$ & $7.5 \pm 0.25$ & $9.0 \pm 0.20$ & $\mathrm{NaHCO} 3$ \\
\hline Total organic carbon (TOC) & $5.0 \mathrm{mg} / \mathrm{L} \dagger$ & $10 \mathrm{mg} / \mathrm{L}$ & Humic acid \\
\hline Turbidity & $15 \pm 1$ NTU $\dagger$ & $100 \mathrm{NTU}$ & $\begin{array}{l}\text { ISO 12103-1, A2 fine test dust } \\
\text { (Power Technology, Burnsville, MN) }\end{array}$ \\
\hline Temperature & $20^{\circ} \mathrm{C} \pm 0.5^{\circ} \mathrm{C}$ & $20^{\circ} \mathrm{C} \pm 0.5^{\circ} \mathrm{C} \neq$ & \\
\hline Total dissolved solids & $480 \mathrm{mg} / \mathrm{L}$ & $15,000 \mathrm{mg} / \mathrm{L}$ & Sea salts, Sigma S9883 or equivalent \\
\hline Disinfectant residue & No detectible disinfectant & No detectible disinfectant & Granular activated charcoal filter \\
\hline Bacteria/virus/cyst & No detectable CFU/100 mL or PFU/L & Challenge level & Refer to test organisms \\
\hline
\end{tabular}

* $\mathrm{CFU}=$ colony forming unit; $\mathrm{PFU}=$ plaque forming unit.

$\dagger$ EPA general test water \#1 prescribes turbidity of $0.1-5.0 \mathrm{NTU}$ and TOC of $0.1-5.0 \mathrm{mg} / \mathrm{L}$.

$\$$ EPA challenge test water $\# 3$ prescribes a temperature of $4^{\circ} \mathrm{C} \pm 0.5^{\circ} \mathrm{C}$.

stocks were prepared by growth on the host bacterium and purified, as previously, and assayed by the double agar overlay method as described earlier. ${ }^{11}$ Cryptosporidium parvum oocysts were obtained from the feces of infected calves and purified by a discontinuous sucrose gradient. ${ }^{14}$ The oocysts were exposed to an ultraviolet (UV)-C light (40 $\mathrm{m} \mu \mathrm{Wsec} /$ $\mathrm{cm}^{2}$ blub) for one hour to inactivate the oocysts to reduce risk of infection during conduct of the tests. Samples of influent $(10 \mathrm{~mL})$ and effluent $(100 \mathrm{~mL})$ were centrifuged at $400 \times \mathrm{g}$ for 15 minutes to pellet the oocysts. After resuspension of the pellet in PBS, the oocysts were counted using a Sotlite hemocytometer (Baxter Healthcare Corp., McGraw Park, IL), with a phase contrast microscope at $400 \times$ magnification.

Water spiking and sampling. Each unit was filled automatically with specified test water when the raw water reservoir reached the refill level specified by the manufacturer. Units were tested in a 16-hour cycle (i.e., minimum of 8 hours off per day) over $\sim 330$ days during which the units produced $20,000 \mathrm{~L}$ representing roughly $110 \%$ of their $18,000 \mathrm{~L}$ design life. Effluent was sampled from the tap of each test unit in accordance with the schedule prescribed by the EPA Protocol. This requires sampling at prescribed percentages of the product's design life. Throughout the test period, average turbidity and bacteria levels were maintained continuously at the prescribed levels. The MS2 virus was spiked into the challenge water at the prescribed concentrations for one complete cycle immediately preceding the taking of a sample and for one complete cycle during the taking of a sample.

Cleaning. In accordance with the manufacturer's instructions and the testing protocol, cleaning of the pre-filter and backwashing of the filter was performed whenever the flow rate fell below $80 \mathrm{~mL} /$ minute. For cleaning, the pre-filter was removed from the raw water reservoir and rinsed by hand in tap water. The filter was cleaned by closing the treated water tap, squeezing the ballcock three times, opening the backwash water tap to let the backwash water flow to waste during a few seconds, and closing the backwash water tap. Once each week during testing, the raw water reservoir was also cleaned with a soft brush or a cloth and rinsed with tap water.

Monitoring and assessment. Quality assurance testing was conducted for the seed organisms under environmental conditions at the beginning and end of each test day to ensure that there was no significant change over the test day. This was done by measuring populations as dispersed by the supply tank at the beginning and end of the 16-hour test day to detect possible incidental effects, such as proliferation, die-off, adsorption to surfaces, etc. The log reduction value (LRV) for each test organism or proxy was determined as follows:

$\mathrm{LRV}=\log _{10}(\mathrm{Cf})-\log _{10}(\mathrm{Cp})$, where $\mathrm{Cf}$ is the feed concentration of the challenge organism or proxy and $\mathrm{Cp}$ is the filtrate concentration of the challenge organism or proxy.

It is noted that to meet the requirements of a microbiological water purifier under the EPA Protocol, the geometric mean of all LRVs must be at least 6 for bacteria, 4 for viruses, and 3 for cysts. Moreover, not more than $10 \%$ of the influent/ effluent sample pairs may deviate from the required LRV by one order of magnitude in the case of bacteria and viruses, and one-half order of magnitude in the case of cysts.

\section{RESULTS}

Microbiologic assessment. Table 2 sets forth the log reduction values at each sampling point for each of the three treatment units for the bacterial, viral, and protozoan cyst test organisms. The mean $\log _{10}$ reduction for all sample pairs over the life of the test protocol was 6.9 for E. coli, 4.7 for $M S 2$, and 3.6 for the C. parvum oocysts. At each sampling point, all three treatment units succeeded in reducing E. coli by at least $4.8 \operatorname{logs}$, MS2 by at least $3.6 \mathrm{logs}$, and Cryptosporidium oocysts by at least $3.1 \mathrm{logs}$.

Flow rate and cleaning. Figure 2 shows the effluent flow rate of $\mathrm{mL} /$ minute for each unit tested over $20,000 \mathrm{~L}(111 \%$ of the $18,000 \mathrm{~L}$ design life). Although the initial flow rates ranged from $200-293 \mathrm{~mL} / \mathrm{minute}$, the rate fell to $129-143 \mathrm{~mL} / \mathrm{minute}$ by $25 \%$ of life $(3,750 \mathrm{~L})$, and to $100-130 \mathrm{~mL} /$ minute by $100 \%$ of life $(18,000 \mathrm{~L})$. Thereafter, flow rates diminished to $60-110$ $\mathrm{L} /$ minute. Over the $18,000 \mathrm{~L}$ design life, the mean flow rates ranged from $132 \mathrm{~mL} /$ minute to $159 \mathrm{~mL} /$ minute, with an overall mean of $146 \mathrm{~mL} /$ minute $(8.8 \mathrm{~L} /$ hour $)$. The average required interval for cleaning to restore flow rate was 11 hours of operation for the filter cartridge and 30 hours for the prefilter.

Filter life. In accordance with the terms of the study protocol, the device was tested through $20,000 \mathrm{~L}$, representing $111 \%$ of its design life. Although flow rates showed some evidence of diminishing over filter life, all three units continued to produce at least $100 \mathrm{~mL} / \mathrm{min}$ through the $18,000 \mathrm{~L}$ design life. There was no evidence of impaired microbiologic performance through 20,000 L of operation.

\section{DISCUSSION}

Results from this assessment indicate that the treatment unit is effective under controlled circumstances in removing a range of microbial indicators of fecal contamination for up to $20,000 \mathrm{~L}$, or roughly $110 \%$ of its design capacity. The average $\log _{10}$ reductions exceeded $6 \operatorname{logs}(99.9999 \%)$ of the test organism for bacteria, 4 (99.99\%) of the test organism for virus, and $3 \operatorname{logs}(99.9 \%)$ of the test organism for protozoan cysts. 
TABLE 2

Summary of log reduction values (LRVs) at each sampling point

\begin{tabular}{|c|c|c|c|c|c|c|c|c|}
\hline \multirow[b]{2}{*}{ Percentage of design life of unit } & \multicolumn{8}{|c|}{ Test point } \\
\hline & $0 \%$ & $25 \%$ & $45 \%$ & $50 \%$ & $60 \%$ & $80 \%$ & $100 \%$ & $110 \%$ \\
\hline Volume passed $(\mathrm{L})$ & 10 & 3,750 & 7,500 & 9,000 & 11,250 & 15,000 & 18,000 & 20,000 \\
\hline \multicolumn{9}{|l|}{ Unit 1} \\
\hline Escherichia coli & $>7.1 *$ & $>8.3$ & $>6.9$ & $>6.9$ & 4.8 & $>7.5$ & $>7.3$ & $>7.6$ \\
\hline MS2 & 3.8 & 5.7 & 4.2 & 3.8 & 3.6 & 4.7 & $>6.0$ & $>6.8$ \\
\hline Cryptosporidium oocysts & $>3.1$ & 3.8 & 3.4 & 3.7 & 3.9 & $>3.7$ & $>3.8$ & $>3.8$ \\
\hline \multicolumn{9}{|l|}{ Unit 2} \\
\hline E. coli & 6.6 & 6.3 & $>6.9$ & 5.8 & 6.1 & $>7.5$ & $>7.3$ & $>7.6$ \\
\hline MS2 & 3.6 & 3.8 & 3.5 & 4.4 & 4.8 & 4.7 & $>6.0$ & $>6.8$ \\
\hline Cryptosporidium oocysts & $>3.1$ & $>3.6$ & 3.4 & 3.4 & 3.5 & $>3.7$ & $>3.8$ & $>3.8$ \\
\hline \multicolumn{9}{|l|}{ Unit 3} \\
\hline E. coli & $>7.1$ & 6.8 & $>6.9$ & 5.4 & 7.0 & $>7.5$ & $>7.3$ & $>7.6$ \\
\hline MS2 & 3.6 & 3.7 & 3.6 & 4.4 & 4.3 & 5.0 & $>6.0$ & $>6.8$ \\
\hline Cryptosporidium oocysts & $>3.1$ & $>3.6$ & 3.6 & 3.2 & 3.9 & $>3.7$ & $>3.8$ & $>3.8$ \\
\hline
\end{tabular}

These LRVs would meet the requirements for a microbial water purifier prescribed by the EPA Protocol.

The treatment unit continued to produce $100-130 \mathrm{~mL}$ of water/minute through $18,000 \mathrm{~L}$, despite increasing the turbidity of the aging water to 15 NTU (150 times the minimum level prescribed by the EPA Protocol) and increasing the total organic carbon level to $5.0 \mathrm{mg} / \mathrm{L}$ (50 times the minimum). This is less than the $150 \mathrm{~mL} /$ minute design rate, but significantly greater than the flow rate from ceramic filters, which are also used in low-income settings. Cleaning was required, but at frequencies that probably would not exceed once per week under real world conditions.

In accordance with the study protocol, testing of the units was terminated after $20,000 \mathrm{~L}$, representing $111 \%$ of design life. At this point, there was no evidence of any failure or diminution of microbiologic performance or flow rate. As the device relies solely on mechanical filtration to remove microbial pathogens from the water, its microbiologic performance should not be impaired by continued use past such levels unless there is a fracture or other failure in the seal that holds the hollow fibers in place. Further testing in the field will help determine the actual longevity of the device and the need for a halogen to control biofilm, which may build up on the hollow fibers over long-term use.

This device is one of the few point-of-use water treatment options designed for routine use in low-income populations that meets the 6-4-3 standard for microbiological water purifiers. Hybrid filters that combine filtration/adsorption with disinfection have been shown to meet the 6-4-3 standard, ${ }^{7,9}$ but

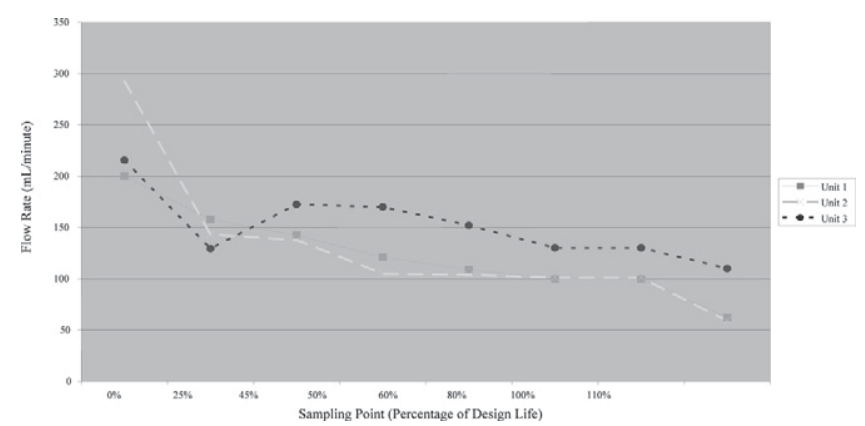

FIGURE 2. Flow rate (mL/minute) of tested units over 20,000 L. these are not fully serviceable in the field and require replacement of consumable components. Sachets combining a flocculant and a disinfectant also have been shown to meet the 6-4-3 standard, ${ }^{15}$ but requires batch treatment of a consumable product. Other common point-of-use water treatment products meet this international standard for bacteria, viruses, or cysts, but not all three. ${ }^{7,9}$

Our results must be interpreted in the context of certain limitations. First, this assessment was undertaken under controlled laboratory conditions, not in the field and not as the unit may actually be used by householders. Second, this evaluation was undertaken using a single aging and challenge water. Although these waters were specifically designed to reflect the key parameters that would challenge filtration devices (and actually exceeded the EPA guidelines for turbidity and TOC), the performance and life of water treatment units can be affected by various chemical and physical conditions that may not be encompassed by these tests. Finally, the 20,000 L evaluation reported here was conducted over just 10 months, less than a quarter of the time a householder might actually be expected to use the device in the field. It is possible that use of the device over longer periods in the tropics could accelerate the growth of biofilm on the hollow fiber membranes and thus impair flow rate or cause premature choking. The manufacturer reports that it has developed a slow-eluting chlorine donor that can be permanently deployed in the unit under the prefilter if field testing shows evidence of adverse impacts because of biofilm. However, this chlorine donor was not incorporated into the units we tested.

We note that as the treatment unit has no residual disinfection, the treated water is immediately susceptible to recontamination, a particular problem in the low-income settings for which it was designed. ${ }^{16}$ Although the manufacturer has designed an alternative configuration that includes a collapsible or rigid container to improve the safe storage of treated water, this will add to the cost. Field testing is underway to investigate the extent to which such recontamination actually occurs when the devices are used by a target population.

Notwithstanding these limitations, this study does establish the basis for further testing in the field. Such testing is necessary to confirm the durability of the system and to identify any potential gaps in performance under actual field conditions. 
It is also important to assess the performance and life of the treatment unit under a wider variety of water conditions and when subjected to less than optimal maintenance.

The ultimate objective of water treatment units such as this, however, is not only to improve water quality but to improve human health. The increasing body of evidence suggesting the potential for household water treatment to dramatically reduce diarrheal morbidity provides good reason to believe that the device can also prevent disease. If this turns out to be the case, then a company that has demonstrated success in the widespread distribution of environmental health products to low-income populations should be encouraged to scale up the intervention on an affordable and sustainable basis.

Received September 22, 2008. Accepted for publication December 14, 2008.

Disclosure: The authors note the following potential conflicts of interest: Thomas Clasen is a member of the Environmental Health Group of the London School of Hygiene and Tropical Medicine, a portion of whose funding is provided by Vestergaard Frandsen S.A., the manufacturer of the LifeStraw Family Filter; Daniel Frauchinger is an employee of Vestergaard Frandsen S.A.; and the costs of the University of Arizona in performing the laboratory work reported in this work were funded by Vestergaard Frandsen S.A.

Authors' addresses: Thomas Clasen, Disease Control and Vector Biology Unit, Department of Infectious and Tropical Diseases, London School of Hygiene and Tropical Medicine, Keppel St., London WC1E 7HT, UK, E-mail: thomas.clasen@lshtm.ac.uk. Jamie Naranjo and Charles Gerba, Department of Soil, Water and Environmental Sciences, University of Arizona, Tucson, AZ 85721. Daniel Frauchiger, Vestergaard Frandsen S.A., Chemin de Messidor 5-7, CH-1006, Lausanne, Switzerland.

\section{REFERENCES}

1. Prüss-Üstün A, Bos R, Gore F, Bartram J, 2008. Safe Water, Better Health: Costs, Benefits and Sustainability of Interventions to Protect and Promote Health. Geneva: World Health Organization.

2. WHO, 2005. World Health Report 2005-Make Every Woman and Child Count. Geneva: The World Health Organization.
3. Blakely T, Hales S, Kieft C, Wilson N, Woodward A, 2005. The global distribution of risk factors by poverty level. Bull World Health Organ 83: 118-126.

4. Fewtrell L, Kaufmann R, Kay D, Enanoria W, Haller L, Colford J, 2005. Water, sanitation, and hygiene interventions to reduce diarrhoea in less developed countries: a systematic review and meta-analysis. Lancet Infect Dis 5: 42-52.

5. Clasen T, Roberts I, Rabie T, Schmidt W, Cairncross S, 2006. Interventions to improve water quality for preventing diarrhoea. The Cochrane Library, Issue 3: Oxford: Update Software.

6. Clasen T, Haller L, Walker D, Bartram J, Cairncross S, 2007. Costeffectiveness analysis of water quality interventions for preventing diarrhoeal disease in developing countries. $J$ Water Health 5: 599-608.

7. Clasen T, 2009. Scaling Up Household Water Treatment among Low-Income Populations. Geneva: World Health Organization.

8. WHO/UNICEF, 2008. Progress in Drinking-water and Sanitation: Special Focus on Sanitation. Geneva:World Health Organization, and New York: United Nations Children's Fund.

9. Sobsey M, 2002. Managing Water in the Home: Accelerated Gains from Improved Water Supply. Geneva: World Health Organization.

10. USEPA, 1987. Guide Standard and Protocol for Testing Microbiological Water Purifiers. Washington, DC: United States Environmental Protection Agency.

11. Grabow WOK, 2001. Bacteriophages: update on application as models for viruses in water. Water $S A$ 27: 251-268.

12. Gerba CP, Naranjo JE, Jones EL, 2008. Virus removal from water by a portable water treatment device. Wilderness Environ Med 19: 45-49.

13. APHA, AWWA \& WEF, 2001. Standard Methods for the Examination of Water and Wastewater. Twentieth edition. Part 2130. Washington, DC: American Public Health Association/American Water Works Association and Water Environment Federation.

14. Arrowood MJ, Sterling CR, 1987. Isolation oocysts and sporozoites using a discontinuous sucrose and isopycnic gradients. J Parasitol 73: 314-319.

15. Reller ME, Mendoza CE, Lopez MB, Alvarez M, Hoekstra RM, Olson CA, Baier KG, Keswick BH, Luby SP, 2003. A randomized controlled trial of household-based flocculant-disinfectant drinking water treatment for diarrhea prevention in rural Guatemala. Am J Trop Med Hyg 64: 411-419.

16. Wright J, Gundry S, Conroy R, 2003. Household drinking water in developing countries: a systematic review of microbiological contamination between source and point-of-use. Trop Med Int Health 9: 106-117. 